\title{
Orientation dependent transverse relaxation in human brain white matter: The magic angle effect on a cylindrical helix
}

\author{
Yuxi Pang, PhD
}

Department of Radiology, University of Michigan, Ann Arbor, MI, USA

\section{Corresponding Author:}

Yuxi Pang, PhD

University of Michigan Hospital

1500 E. Medical Center Dr., UH B2 RM A205F

Ann Arbor, MI 48109-5030, USA

Tel: $\quad$ 734-232-6585

Fax: $\quad$ 734-764-2412

Email: $\quad$ yuxipang@umich.edu

Twitter: @yuxipang

ORCID iD: Yuxi Pang (https://orcid.org/0000-0001-5039-0236)

Running Title: Orientation dependent transverse relaxation in white matter

Word Count: 5149 


\begin{abstract}
Purpose: To overcome limitations of prior orientation-dependent $R_{2}$ and $R_{2}^{*}$ formalisms in white matter (WM) with a novel framework based on magic angle effect.

Methods: A cylindrical helix model was developed embracing both anisotropic rotational and translational diffusions of ordered water in WM, with the former characterized by an axially symmetric system. Both $R_{2}$ and $R_{2}^{*}$ were divided into isotropic $\left(R_{2}^{i}\right)$ and anisotropic parts, $R_{2}^{a} * f\left(\varepsilon-\varepsilon_{0}, \alpha\right)$, with $\alpha$ denoting a funnel opening angle and $\varepsilon_{0}$ an orientation $(\varepsilon)$ offset relative to DTI-derived primary diffusivity direction. The proposed framework (Fit A) was compared with prior model without $\varepsilon_{0}$ (Fit B) and applied to published $R_{2}$ and $R_{2}^{*}$ in WM of underdeveloped, healthy, and diseased conditions. Goodness of fit was characterized by rootmean-square error (RMSE). F-test and Pearson correlation coefficient (PCC) were used with statistical significance set to $P \leq .05$.
\end{abstract}

Results: Fit A significantly outperformed Fit B as demonstrated by reduced RMSEs in myelin water (i.e., 0.349 vs. 0.724 ). The fitted $\varepsilon_{0}$ was in good agreement with the calculated $\varepsilon_{0}$ from DTI directional diffusivities. Significant positive $\left(R_{2}^{i}\right)$ and negative $\left(\alpha\right.$ and $\left.R_{2}^{a}\right)$ correlations were found with aging (demyelination) in adults while $\varepsilon_{0}$ showed a weak positive correction (PCC $=0.11, P=.28$ ). Compared to those from healthy adult WM, the fits of $R_{2}^{i}, R_{2}^{a}$, and $\alpha$ from neonates were considerably reduced but $\varepsilon_{0}$ increased, consistent with limited myelination.

Conclusion: The developed framework can better characterize anisotropic transverse relaxation in WM, shedding new light on myelin microstructural alterations at the molecular level.

Keywords: anisotropic transverse relaxation, cylindrical helix, magic angle effect, anisotropic rotational diffusion, anisotropic translational diffusion, white matter. 


\begin{abstract}
Abbreviations: AR, angular resolution; CPMG, Carr-Purcell-Meiboom-Gill; CNT, control; DTI, diffusion tensor imaging; FA, fractional anisotropy; IE, intra- and extra-cellular; MAE, magic angle effect; MS, multiple sclerosis; PCC, Pearson correlation coefficient; qMT, quantitative magnetization transfer; RDC, residual dipolar coupling; RMSE, root-mean-square error; SBL, siblings; WM, white matter
\end{abstract}

\title{
1 | INTRODUCTION
}

Magic angle effect (MAE) is a well-known phenomenon of orientation-dependent nuclear transverse relaxation in MR imaging of highly organized biological tissues. ${ }^{1,2}$ The origin of this effect has been ascribed to restricted water molecular reorientations in aqueous heterogeneous systems. $^{2-4}$ Early experience on MAE in biological systems comes from NMR study of hydrated Bovine Achilles tendon, ${ }^{5}$ where the direction $(\theta)$ of an apparent residual dipolar coupling (RDC) from restricted water in collagenous fibers is found predominantly parallel to the primary axis of tendon. This is because that water molecules buried inside triple-helical microstructures of collagen can hardly undergo any rotational motions other than preferentially around the principal axis of the molecule. ${ }^{2,6}$

The water proton transverse relaxation rate (i.e., $R_{2}=1 / T_{2}$ ) in ordered microstructures can be divided into an isotropic and an anisotropic component, with the latter characterized by an orientation-dependent factor $\left(3 \cos ^{2} \theta-1\right)^{2}$. If $\theta$ is inclined at the "magic angle" of $54.7^{\circ}$, relative to the applied magnetic field $B_{0}$, this spatial factor will be zero leading to a lengthened $T_{2}$. As a result, tendon may become conspicuous at the magic angle in clinical $\mathrm{T} 2 \mathrm{~W}$ images otherwise undetectable due to an intrinsic ultra-short $T_{2} \cdot{ }^{1}$ It should be emphasized that this 
standard MAE function implicitly assumes that all restricted water be arranged uniformly along the same direction in highly organized tissues, which might not be valid in some cases. ${ }^{7,8}$ Although it has been long before documented in the peripheral nervous system, ${ }^{9}$ MAE still remains elusive in the human brain white matter (WM) despite that extensive research has been performed in the past two decates. ${ }^{10-12}$

Based on the standard MAE function, $R_{2}$ is expected to be decreased if an axonal fiber angle $\theta$ increases from $0^{\circ}\left(R_{2 \|}\right)$ to $90^{\circ}\left(R_{2 \perp}\right)$, i.e., $R_{2 \|}>R_{2 \perp}$; yet the measured $R_{2 \|}$ appears always smaller than $R_{2 \perp} \cdot{ }^{12-15}$ Further, MAE is assumed exclusively involving collagen that is hardly found in the human brain parenchyma. ${ }^{13}$ Hence, MAE has not been considered as a potential anisotropic $R_{2}$ relaxation mechanism. Instead, an orientation-dependent $R_{2}$ formalism, i.e., $A_{1}+A_{2} \sin ^{2} \theta+A_{3} \sin ^{4} \theta$, was proposed based on a model of cylindrical magnetic field perturbers. ${ }^{11}$ The $\sin ^{2} \theta$ term predicts T2W signal decay due to the interaction between myelininduced susceptibility differences and applied imaging field gradients, and the $\sin ^{4} \theta$ term reflects the diffusion-induced magnetization dephasing due to susceptibility-based inhomogeneous fields.

Similarly, the observed $R_{2 \|}^{*}$ from several gradient-echo studies is also unexpectedly smaller than $R_{2 \perp}^{*} \cdot{ }^{16-18}$ The standard MAE is just incompatible with the fact $R_{2 \|}^{*}<R_{2 \perp}^{*}$ in WM even though it can partially account for the measured temperature-dependent $R_{2}^{*}$ orientational anisotropy. ${ }^{19}$ Nonetheless, an orientation-dependent $R_{2}^{*}$ model was proposed based on the same "cylindrical perturbers" model, ${ }^{18,20}$ and formulated as $B_{1}+B_{2} \cos 2 \theta+B_{3} \cos 4 \theta$. While the $\cos 2 \theta$ term characterizes susceptibility effect induced by cylindrical structures of WM fibers, the $\cos 4 \theta$ term is ascribed to the effect of susceptibility anisotropy originated from myelin 
unique microstructures. ${ }^{19}$ The same formalism used for quantifying $R_{2}$ orientational anisotropy was also suggested for orientation-dependent $R_{2}^{*}$ based on a hollow cylinder fiber model. ${ }^{21,22}$

As pointed out recently, ${ }^{12,} 23$ both $R_{2}$ and $R_{2}^{*}$ formalisms abovementioned are mathematically equivalent albeit with different coefficients $A_{i}$ and $B_{i} \quad(i=1,2,3)$. These formalisms have been widely used in anisotropic transverse relaxation studies of WM in vivo, for instance, orientation-dependent myelin water imaging, ${ }^{14} R_{2}^{*}$ mapping in neonates ${ }^{24}$ and people with multiple sclerosis (MS). ${ }^{25}$ Despite widespread adoptions, these proposed models have some major limitations bearing significant implications when interpreting the fitted model parameters. First, the coefficients $A_{i}$ (or $B_{i}$ ) are not mutually independent as $\sin ^{4} \theta$ (or $\cos 4 \theta$ ) can be expressed by $\sin ^{2} \theta$ (or $\cos 2 \theta$ ) and vice versa; ${ }^{12,} 14$ thus, any suggested biophysical interpretations of the estimated parameters are ambiguous. Second, a noticeable angle shift in $R_{2}$ or $R_{2}^{*}$ orientation dependence profile has not been accounted yet. This angle shift became exacerbated when diffusion fractional anisotropy (FA) decreased as appeared in neonatal WM. ${ }^{24}$ Thus, these two major drawbacks, if not rectified, would lead to different conclusions depending on seemingly different but mathematically identical formulations, ${ }^{12,} 23$ ultimately compromising the underlying myelin specificity of anisotropic $R_{2}$ or $R_{2}^{*}$ in WM.

In literature, ${ }^{26}$ an advanced Raman scattering microscopic imaging study has demonstrated that water molecules close to phospholipid bilayer surface are perpendicular to rather than parallel along an axon primary axis. As recognized long before and demonstrated recently, ${ }^{5,7,8}$ an anisotropic transverse relaxation profile can be altered significantly depending on how organized are the relevant RDCs from either ordered water or other restricted proton groups. For instance, a recent quantitative magnetization transfer (qMT) study embraced a cylinder model where the effective RDCs of methylene $(\mathrm{CH} 2)$ groups of semisolid lipid's long 
tails were considered as concentrically organized. ${ }^{27}$ As a result, the orientation-dependent absorption linewidths (or $\mathrm{T}_{2}^{b}$ ) of $\mathrm{CH} 2$ groups of semisolid macromolecules in WM were successfully accounted for to a large extent.

To date, an axon fiber orientation $(\theta)$ in vivo in an image voxel is exclusively deduced from DTI, ${ }^{14,28}$ normally referred to the direction of axial diffusivity $\left(D_{\|}\right)$relative to $B_{0}$ given that diffusion can be modeled as a zeppelin tensor assuming $D_{\|}>D_{\perp}$ (radial diffusivity). This standard practice seems valid only for a "stick" tensor (i.e., $D_{\perp}=0$ ) as far as an anisotropic diffusion is concerned. For a general case (i.e., $D_{\perp} \neq 0$ ), $D_{\|}$will contain not only an anisotropic but also an isotropic diffusion component; therefore, the direction of $D_{\|}$may deviate from the actual axon fiber direction with which an anisotropic $R_{2}$ or $R_{2}^{*}$ is directly associated. This general case most likely contributed to an obvious angle shift in the measured $R_{2}$ and $R_{2}^{*}$ orientation dependence profiles that had never been accounted before. ${ }^{14,24}$ Together with the limitations of prior orientation-dependent $R_{2}$ and $R_{2}^{*}$ formalisms, there is apparently an unmet need for developing a new biophysical model to intrinsically connect an anisotropic transverse relaxation in WM with the underlying highly organized myelin microstructure.

This work thus aimed to develop a theoretical framework by generalizing the standard MAE function and considering the DTI-related angle shift for better characterizing anisotropic $R_{2}$ and $R_{2}^{*}$. The developed framework was applied to previously reported anisotropic $R_{2}$ and $R_{2}^{*}$ in WM from the human brain of neonates, healthy and diseased adults in vivo at 3T. The fitting results demonstrate that the proposed framework can better characterize the documented anisotropic transverse relaxation profiles, thereby providing further insights into WM microstructural alterations at the molecular level.

\section{2 | THEORY}


As revealed by an advanced Raman scattering microscopic imaging study, ${ }^{26}$ water molecules close to the hydrophilic surface of phospholipid bilayers are highly organized (on the time scales of $10^{-14} \mathrm{~s}$ ) as schematically depicted in Figure 1A, with water principal symmetry axis (green dashed line) along the direction normal to the bilayer as highlighted in Figure 1B. Consequently, an effective internuclear dipolar interaction of dynamically ordered water, denoted by $\langle\mathrm{H}-\mathrm{H}\rangle$, is not averaged to zero, leading to a residual dipolar coupling (RDC) perpendicular to the lipid's long hydrophobic chains. In other words, the reorientation of ordered water is most limited around an axis along the axon but less restricted around an axis perpendicular to the surface largely depending on the local environments (or membrane layers). Within the phospholipid bilayers, the lipid molecule not only diffuses laterally but also rotates rapidly about its long axis. $^{29,30}$ As a result, an effective RDC of multiple methylene $(\mathrm{CH} 2)$ groups on the long chains becomes orthogonal to the surface ${ }^{27}$ and thus perpendicular to the RDC of the ordered water. Practically, an ensemble of RDCs from either restricted water molecules or $\mathrm{CH} 2$ groups can be considered as concentrically distributed around an axon; thus, they can be characterized by an axially symmetric system as demonstrated in Figures $1 \mathrm{C}$ and 1D, with the axon primary direction $(\vec{n})$ respectively parallel to and perpendicular to the main magnetic field $B_{0}$.

Based on this axially symmetric model, an effective RDC within an image voxel will become larger when $\vec{n}$ is perpendicular than parallel to $B_{0}$. Proportional to the variance of RDC, 5 the transverse relaxation rate follows the same trend of RDC orientation dependences as previously reported $R_{2 \perp}^{*}>R_{2 \|}^{*} 15-17,20,23,28$ or $R_{2 \perp}>R_{2 \|}{ }^{12-15}$. Typically, an axonal fiber tract in an image voxel contains a bundle of axons potentially with different diameters, and each axon is encased spirally by multiple layers of plasma membranes as schematically shown in Figure $3 \mathrm{C} .^{31}$ Nevertheless, a representative axon can be considered, with an effective radius $r$ depending on 
the degree of myelination. To generalize the axially symmetric (or funnel) model as shown in Fig. 1C, the angle $(\alpha)$ between the direction of $\langle\mathrm{H}-\mathrm{H}\rangle$ and $\vec{n}$ will be considered adjustable as depicted in Figure 2A, i.e., not limited only to $90^{\circ}$. When changing from a $2 \mathrm{D}$ planar view into a 3D spatial perspective, as illustrated in Figure 2B, a stack of identical funnels can be conceptualized in space along $\vec{n}$. These stacked funnels can be well represented by a representative axially symmetric model (Fig. 2A); however, it is much enlightening to create a mathematically equivalent cylindrical helix as depicted in Figure $2 \mathrm{C}$, where the $\langle\mathrm{H}-\mathrm{H}\rangle$ vector makes a constant angle $\alpha$ to $\vec{n}$ along the circular helix trajectory.

This circular helix trajectory can be parameterized with $r$ and a helix pitch of $2 \pi d$, i.e., $[x(t), y(t), z(t)]=[r \cos t, r \sin t, d t]$ with $t$ denoting time. Suppose there are $N$ turns of helix circles, if unfolded, the total circumference length and height will be $2 \pi r N$ (adjacent side) and $2 \pi d N$ (opposite side) respectively, which can form two sides of a triangle in which the hypotenuse (or tangent) line makes an angle $\alpha$ to the opposite side. Thus, a constant angle $\alpha$, between any tangent line and $\vec{n}$, can be calculated using an equation of $\tan \alpha=r / d$. While the membrane microstructure should remain constantly stable (i.e., a fixed $d$ ) in normal physiological conditions, an effective $r$ could change depending on an extent of myelination such as neuronal development or demyelination due to aging and pathologies. Accordingly, the angle $\alpha$ could be regarded as a potential myelination biomarker reflecting the changes of an effective helix radius $r$.

Considering a time-averaged rather than ensemble-averaged RDC with a spiraling $<\mathrm{H}-$ $\mathrm{H}>$ vector, the proposed cylindrical helix model essentially comprises both an anisotropic rotational motion around $\vec{n}$ and an anisotropic translational diffusion along $\vec{n}$. Instead of using $D_{\|}$ direction as an anisotropic orientation reference as schemed in Figure 2D, the direction of the 
vector difference between $D_{\|}$and $D_{\perp}$ will be considered herein as an effective anisotropic translational diffusion along $\vec{n}$. This is because $D_{\|}$reflects not only an anisotropic but also an isotropic translational diffusion, and $D_{\perp}$ is taken herein as an isotropic translational diffusion contribution. The amount, but not the direction, of an anisotropic translational diffusion has been discussed in the literature. ${ }^{32}$ Accordingly, an offset angle $\varepsilon_{0}$ has been introduced in this work for modeling the reported anisotropic $R_{2}$ and $R_{2}^{*}$ profiles in WM. As a comparison, this DTI-related $\varepsilon_{0}$ can be calculated by $\tan ^{-1}\left(D_{\perp} / D_{\|}\right)$if both $D_{\|}$and $D_{\perp}$ are available, and it increases almost linearly from $0^{\circ}$ to $45^{\circ}$ as the translational diffusion changes from being ideally anisotropic (i.e., $\left.D_{\perp}=0\right)$ to isotopic (i.e., $D_{\perp}=D_{\|}$).

As shown in Figure $3 \mathrm{~A}$, when $\vec{n}$ deviates from $B_{0}$ by an angle $\varepsilon$, a typical $\langle\mathrm{H}-\mathrm{H}\rangle$ vector can be identified by an angle $\theta$ to $B_{0}$ and an angle $\alpha$ to $\vec{n}$. For this axially symmetric model, $\alpha$ and $\varepsilon$ are constant but $\theta$ and $\varphi$ (azimuthal angle) become spatially or temporally varied. To calculate an effective anisotropic transverse relaxation rate, the spatial factor $\left\langle\left(3 \cos ^{2} \theta-1\right)^{2}\right\rangle$ ought to be evaluated. Herein, angle brackets stand for an ensemble or time average. ${ }^{5,8,} 33$ According to the spherical law of $\operatorname{cosines,~the~term~} \cos \theta$ in the standard MAE function can be recast into the expression of $\cos \alpha \cos \varepsilon+\sin \alpha \sin \varepsilon \cos \varphi$. After substituting the $\cos \theta$ term and averaging over all $\varphi$ values ranging from 0 to $2 \pi$, the spatial factor $\left\langle\left(3 \cos ^{2} \theta-1\right)^{2}\right\rangle$ can be written as a compact function $f(\alpha, \varepsilon)$ as shown in Eq. 1, referred to as a generalized MAE function in the literature. ${ }^{8}$

$$
\begin{aligned}
& f(\alpha, \varepsilon)=\frac{1}{4}\left(3 \cos ^{2} \alpha-1\right)^{2}\left(3 \cos ^{2} \varepsilon-1\right)^{2}+\frac{9}{8}\left(\sin ^{4} \alpha \sin ^{4} \varepsilon+\sin ^{2} 2 \alpha \sin ^{2} 2 \varepsilon\right) \\
& f(\alpha, \varepsilon)=\frac{1}{512}\left(a_{0}+a_{1} \cos 2 \varepsilon+a_{2} \cos 4 \varepsilon\right)
\end{aligned}
$$

Note, any trigonometric term containing either $\langle\cos \varphi\rangle$ or $\langle\cos 2 \varphi\rangle$ appeared in the expanded function has been averaged out. If angle $\alpha$ is equal to $0^{\circ}, f(\alpha, \varepsilon)$ will restore the standard MAE 
function $\left(3 \cos ^{2} \varepsilon-1\right)^{2}$. On the other hand, a little-known formalism, i.e., $1-3 \sin ^{2} \varepsilon+$ $(27 / 8) \sin ^{4} \varepsilon$, will be attained when $\alpha=90^{\circ} .{ }^{7}$

Eq.1 can also be rearranged in terms of $\cos 2 \varepsilon$ and $\cos 4 \varepsilon$ as shown in Eq. 2, with the coefficients

$$
a_{0}=81 \cos 4 \alpha+156 \cos 2 \alpha+467
$$$$
a_{1}=180 \cos 4 \alpha+432 \cos 2 \alpha+156
$$

and $a_{2}=315 \cos 4 \alpha+180 \cos 2 \alpha+81$. This is the original functional form used by Berendsen in his seminal paper. ${ }^{5}$ Obviously, these coefficients $a_{i}(i=0,1,2)$ are mutually dependent. As revealed in the literature, ${ }^{12}$ Eq. 2 can also be written as a superposition of the $\sin ^{2} \alpha$ and $\sin ^{4} \alpha$ terms plus a constant, which have been widely used for characterizing orientation-dependent $R_{2}$ and $R_{2}^{*}$ in WM, but without considering an angle shift $\varepsilon_{0}$ for DTI-derived orientations. ${ }^{11,12,14,18,19,21}$

\section{3 | METHODS}

Unless otherwise specified, all orientation-dependent transverse relaxation profiles, identified by letters in an alphabetical order (see Tables 1 and 3), were extracted from image-based graphs in previous publications using a free online tool (www.graphreader.com), and replotted in the figures (black triangles) herein. The orientations $(\varepsilon)$ associated with the measured $R_{2}$ and $R_{2}^{*}$ were determined by the primary diffusivity direction $\left(\vec{e}_{1}\right)$ relative to the direction of the main magnetic field $\vec{B}_{0}$, i.e., $\cos \varepsilon=\left(\vec{e}_{1} \cdot \vec{B}_{0}\right) /\left(\left|\vec{e}_{1}\right| \cdot\left|\vec{B}_{0}\right|\right)$. These estimated $\varepsilon$ values were sorted by increasing angles from $0^{\circ}$ to $90^{\circ}$, and further averaged within a predefined interval or angular resolution (AR). $R_{2}$ and $R_{2}^{*}$ mappings were acquired using conventional Carr-Purcell-MeiboomGill (CPMG) spin-echo and gradient-echo sequences, respectively. The original relaxation data were acquired from whole brain WM on 3T MR systems, following the ethical guidelines as stated in the original publications, with the written informed consents signed by participants and approvals of local Institutional Review Boards (IRBs).

\subsection{Compartmental anisotropic $\boldsymbol{R}_{2}$ from myelin water imaging (Group A)}


Anisotropic $R_{2}$ of myelin water and intra- and extra-cellular (IE) water were respectively retrieved from Figures $7 \mathrm{~A}$ and $7 \mathrm{~B}$ in a recent study of orientation-dependent myelin water imaging, ${ }^{14}$ and replotted in Figures $4 \mathrm{~A}$ and $4 \mathrm{~B}$ herein. These orientation-resolved $\left(\mathrm{AR}=5^{\circ}\right)$ compartmental $R_{2}$ values were obtained from eight healthy volunteers with a mean age of 26 years (range $=21-33$ years) as listed in Table 1.

Average axial $\left(D_{\|}\right)$and radial $\left(D_{\perp}\right)$ diffusivities in WM for an age-matched group at $3 \mathrm{~T}$ were retrieved from Table 1 in another prior publication and relisted herein. ${ }^{34}$ The corresponding DTI-related $\varepsilon_{0}$ (as defined in Figure 2D) and its uncertainty $\Delta \varepsilon_{0}$ were respectively calculated as $\tan ^{-1}(1 / \rho)$ and $\Delta \rho /\left(1+\rho^{2}\right)$, with $\rho=D_{\|} / D_{\perp}$ and $\Delta \rho$ derived from $\Delta D_{\|}$and $\Delta D_{\perp}$ following the basic error propagation rules. ${ }^{35}$ Diffusion fractional anisotropy (FA) was also provided according to

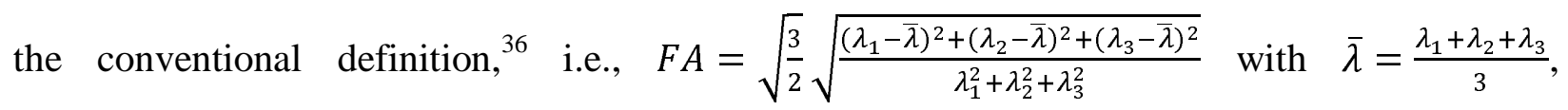
assuming that $\lambda_{1}=D_{\|}, \lambda_{2}=\lambda_{3}=D_{\perp}$.

\subsection{Anisotropic $R_{2}^{*}$ from neonates and adults (Group B)}

A comparative study of anisotropic $R_{2}^{*}$ was performed on two identical MR systems located in two countries, ${ }^{24}$ with eight term neonates (mean age $=40.4 \pm 1.1$ weeks) imaged in China, and five healthy adult volunteers (mean age $=31.2 \pm 8.3$ years) scanned in Canada. The reported anisotropic $R_{2}^{*}\left(\mathrm{AR}=0.33^{\circ}\right)$ values were retrieved respectively from Figures $3 \mathrm{C}$ (neonates) and $4 \mathrm{C}$ (adults) in the original paper and replotted in Figures 5A and 5B herein. The directional diffusivities (i.e., $D_{\|}$and $D_{\perp}$ ) and FA were also retrieved from the original study, from which the corresponding DTI-related $\varepsilon_{0}$ was calculated as mentioned above.

\subsection{Anisotropic $R_{2}^{*}$ from people with MS and two controls (Group C)}

A clinical study of anisotropic $R_{2}^{*}$ in WM was conducted among people with multiple sclerosis (MS, n=39; mean age $=49.7 \pm 10.1$ years), their age-matched asymptomatic siblings $(\mathrm{SBL}, \mathrm{n}=28)$ 
and age-matched unrelated healthy controls $(\mathrm{CNT}, \mathrm{n}=27) .{ }^{25}$ The measured anisotropic $R_{2}^{*}$ profiles $\left(\mathrm{AR}=5^{\circ}\right)$ of each subject from three groups were retrieved from Supporting Information in the original paper, and the group-average $R_{2}^{*}$ profiles were replotted in Figure 6A herein.

\section{4 | Anisotropic $R_{2}^{b}$ deduced from semisolid macromolecules in WM (Group D)}

A quantitative magnetization-transfer (qMT) neuro imaging was performed on 7 healthy subjects (mean age $=26$ years, range $=19-33$ years $).{ }^{27}$ Based on a two-compartment model comprising a liquid pool of free water and a semisolid pool of macromolecules, the system characteristic MT parameters were determined. The observed average orientation-dependent transverse relaxation time $T_{2}^{b}$ profiles of semisolid macromolecules in WM were retrieved from Figure 5 (with different FA thresholds) in the original paper and replicated in Figure 7 in this work.

\section{5 | Nonlinear least-squares curve fittings}

A Levenberg-Marquardt nonlinear least-squares optimization method, implemented in a publicly available IDL script (http://purl.com/net/mpfit), ${ }^{37}$ was used for modeling anisotropic $R_{2}$ and $R_{2}^{*}$ profiles based on the proposed anisotropic relaxation model $\left(R_{2,1}\right)$ in Eq. 3, herein referred to as "Fit A".

$$
\begin{aligned}
& R_{2,1}=R_{2}^{i}+R_{2}^{a} * f\left(\alpha, \varepsilon-\varepsilon_{0}\right) \\
& R_{2,2}=b_{0}-b_{1} \cos 2 \varepsilon+b_{2} \cos 4 \varepsilon
\end{aligned}
$$

There were four model parameters and one independent variable $(\varepsilon)$ in this model, i.e., $R_{2}^{i}$ and $R_{2}^{a}$ denoting orientation independent and dependent contributions, an angle $\alpha$ for a particular ordered water arrangement, and an angle offset (or a phase shift) $\varepsilon_{0}$ relative to $D_{\|}$as shown in Figure 2D. The same data from Groups A and B were also fitted using a previously developed model $R_{2,2}$ as written in Eq. 4 (labeled as "Fit B"), without a phase shift. It should be emphasized 
that when "Fit B" includes $\varepsilon_{0}$, it will become equivalent to "Fit A" albeit with different model parameters.

The curve fittings were unweighted and constrained, initiating with five sets of different values within different constraints. ${ }^{38}$ The fit parameters were constrained as follows: $R_{2}^{i}=b_{0}=[0$, 100] $(1 / \mathrm{s}) ; R_{2}^{a}=b_{1}=[0,50](1 / \mathrm{s}) ; \alpha=\left[0^{\circ}, 90^{\circ}\right] ; \varepsilon_{0}=\left[-45^{\circ}, 45^{\circ}\right] ;$ and $b_{2}=[0,10](1 / \mathrm{s})$. Goodness of fit was represented by root-mean-square error (RMSE), and its statistical significance was assessed by an $F$-test when comparing two fitting models. A $P$-value was derived from $F$ distributions with significance indicated by $P \leq .05$.

All the reported fitting results (mean \pm standard deviation) were obtained from "Fit A", which are tabulated in Tables 1 and 3 and further plotted using solid lines in the presented graphs herein. Pearson correlation coefficients (PCC) are given in Table 2 between the fits from Group $\mathrm{C}$ and the age of each participant from individual or combined subgroups. The statistical significance of observed linear correlations was also set to $P \leq .05$. All image and data analysis were performed using customized code written in IDL 8.8 (Harris Geospatial Solutions, Broomfield, CO, USA).

\section{4 | RESULTS}

\subsection{A theoretical orientation dependent function $f(\alpha, \varepsilon)$}

Figure 3B presents five theoretical $f(\alpha, \varepsilon)$ profiles with different $\alpha$ ranging from $0^{\circ}$ (red) to $90^{\circ}$ (magenta). Compared to that with $\alpha=0^{\circ}$, the degrees of orientation dependences are considerably reduced with $\alpha \neq 0^{\circ}$. Additionally, an orientational anisotropy, defined as $f\left(\alpha, 0^{\circ}\right)$ $f\left(\alpha, 90^{\circ}\right)$, can be reversed with a varying $\alpha$. More importantly, $f(\alpha, \varepsilon)$ can only become zero with $\alpha=0^{\circ}$; in other words, the "magic angle" does not exist and $f(\alpha, \varepsilon)$ is always larger than 
zero when $\alpha \neq 0^{\circ}$. Figure 3D plots an angle $\alpha$ as a function of $r / d$ for the cylindrical helix model. Considering a fixed $d$, the angle $\alpha$ will increase asymptotically to $90^{\circ}$ as $r$ grows.

\section{2 $\mid$ Anisotropic $R_{2}$ from myelin water imaging (MWI)}

Figure 4 demonstrates that Fit A (red solid lines) significantly outperformed Fit B (blue dashed lines) for characterizing anisotropic $R_{2}$ profiles (black triangles) of myelin water (Fig. 4A) and intra- and extra-cellular (IE) water (Fig. 4B) from Group A. ${ }^{14}$ These improvements were quantified by reduced fitting residuals (i.e., $\Delta R_{2}=$ Fit-Exp) as shown in Figs. $4 \mathrm{C}$ and $4 \mathrm{D}$. Specifically, the calculated RMSEs from Fit A, compared to those from Fit B, decreased almost half for myelin water (i.e., 0.350 vs. $0.724, P<.01$ ) and IE water (i.e., 0.011 vs. $0.021, P<.01$ ).

Between two partitioned water pools, the fitted $R_{2}^{i}(1 / \mathrm{s})$ (i.e., $76.3 \pm 0.6$ vs. $13.8 \pm 0.0$ ) and $R_{2}^{a}(1 / \mathrm{s})$ (i.e., $16.1 \pm 0.8$ vs. $\left.1.0 \pm 0.0\right)$ appeared markedly different although $\alpha$ (i.e., $67.0 \pm 0.6^{\circ}$ vs. $69.0 \pm 0.2^{\circ}$ ) and $\varepsilon_{0}$ (i.e., $15.2 \pm 1.1^{\circ}$ vs. $16.1 \pm 0.9^{\circ}$ ) were similar. As expected, the DTI-derived $\varepsilon_{0}$ (i.e., $16.7 \pm 6.1^{\circ}$ ) in highly anisotropic (i.e., $\mathrm{FA}=0.60 \pm 0.12$ ) WM was comparable with the fitted $\varepsilon_{0}$ from the measured anisotropic $R_{2}$ profiles.

\subsection{Anisotropic $R_{2}^{*}$ in WM from neonates and adults}

The observed $R_{2}^{*}$ profile of neonates (Figure 5A) was considerably different from that of adults (Figure 5B). Fit A significantly outperformed Fit B for neonates (Figure 5C) but this is not the case for adults (Figure 5D). With respect to those from adults, the fits from neonates were smaller for $R_{2}^{i}(1 / \mathrm{s})$, i.e., $7.2 \pm 0.1$ vs. $16.1 \pm 0.1 ; R_{2}^{a}(1 / \mathrm{s})$, i.e., $1.3 \pm 0.1$ vs. $8.7 \pm 0.2$ and $\alpha\left(^{\circ}\right)$, i.e., 38.1 \pm 0.4 vs. $70.5 \pm 0.2$, but became larger for $\varepsilon_{0}\left(^{\circ}\right.$ ), i.e., $38.5 \pm 0.3$ vs. $22.7 \pm 0.7$ (vertical dashed green lines). Although the fitted and DTI-derived $\varepsilon_{0}\left(^{\circ}\right)$ were almost the same for adults, the former was relatively larger than the latter for neonates, i.e., $38.5 \pm 0.3$ vs. $32.1 \pm 1.1$, which could be partially caused by an inaccurate replication of the scattered $R_{2}^{*}$ data from the original graph. 
Nonetheless, WM of neonates appeared less anisotropic than those of adults as indicated by the reported FA, i.e., $0.31 \pm 0.01$ vs. $0.53 \pm 0.03$.

\subsection{Anisotropic $R_{2}^{*}$ in WM with multiple sclerosis (MS)}

Figure 6A plots the average $R_{2}^{*}$ orientation-dependent profiles for multiple sclerosis (MS, red), age-matched siblings (SBL, green) and unrelated healthy control (CNT, blue) groups, with comparable fits of $\alpha$ and $\varepsilon_{0}$ as imprinted by colors. The fits of $R_{2}^{i}$ and $R_{2}^{a}$, with the lowest in the

MS subgroup, are shown in Figure 6B. Scatterplots between the ages and the fits of $R_{2}^{i}$ (Fig. 6C), $R_{2}^{a}$ (Fig. 6D), $\alpha$ (Fig. 6E) and $\varepsilon_{0}$ (Fig. 6F) from people in subgroups are presented with a $95 \%$ confidence ellipse overlaid. Compared to those from two controls, $R_{2}^{i}$ and $R_{2}^{a}$ from MS subgroup appeared decreased but $\varepsilon_{0}$ increased.

Linear regression lines from combined subgroups were also included, which highlighted significant positive $\left(R_{2}^{i}\right)$ and negative $\left(R_{2}^{a}\right.$ and $\left.\alpha\right)$ correlations (Table 2) between the aging (or demyelination) and the fits. The fitted $\varepsilon_{0}$ somewhat followed the trend of $R_{2}^{i}$. Generally, an older people most likely possessed increased $R_{2}^{i}$ and $\varepsilon_{0}$ but decreased $R_{2}^{a}$ and $\alpha$, largely in accordance with what was found in neonates with underdeveloped WM.

\section{5 | Anisotropic $T_{2}^{b}$ of WM macromolecules from quantitative MT}

The fitted qMT-derived $T_{2}^{b}$ (i.e., $1 / R_{2}$ ) profiles of semisolid macromolecules in WM from image voxels with FA limited to the ranges of $[0.7,1.0),[0.5,0.7)$ and $[0.3,0.5)$ are shown respectively in Figures 7A-7C. When FA threshold was lowered, the observed $T_{2}^{b}$ orientational anisotropy became increasingly reduced as demonstrated by the increased fitted $R_{2}^{i}$ and $\alpha$ and decreased fitted $R_{2}^{a}$ and $\varepsilon_{0}$ as tabulated in Table 3.

When FA>0.7, the fitted $\alpha$ appeared surprisingly comparable to that (Fig. 5B) found in healthy adults (with similar ages) even though they were attained from distinct proton groups 
using drastically different measurement methods. The fitted $R_{2}^{i}$ and $R_{2}^{a}$ were respectively $54.4 \pm 2.1 \times 10^{3}(1 / \mathrm{s})$ and $42.5 \pm 3.6 \times 10^{3}(1 / \mathrm{s})$, consistent well with the semisolid nature of WM macromolecules. Had the angle $\alpha$ been fixed to $90^{\circ}$ and $\varepsilon_{0}$ set to zero as assumed in the original paper, an alternative curve fitting, labeled as qMT in a blue dashed line (Fig. 7A), would have become markedly inferior to Fit A, as indicated by a significantly increased RMSE (i.e., 2.46 vs. $0.79, P<.01)$.

\section{5 | DISCUSSION}

\subsection{A cylindrical helix model for magic angle effect (MAE) in WM}

Water molecular diffusion in ordered biological tissues comprises not only the translational but also the rotational degrees of freedom. Both types of molecular motions in the human brain white matter (WM) can be categorized into an isotropic and an anisotropic component, with the latter directly associated with the underlying highly organized myelin microstructures. Although diffusion fractional anisotropy (FA) has been long before known proportional to myelin water fraction (i.e., multicomponent $T_{2}$ relaxation), ${ }^{34}$ these two distinct quantitative MR measures have never been considered all together in a concerted system. This work has developed a new model intrinsically connecting these two markedly different anisotropic molecular motions. Although it was conceived out of practical consideration, the proposed geometry of lipid bilayer membrane dose not conflict with the existing biophysical theories. ${ }^{39}$ The proposed cylindrical helix model is unique in that the residual dipolar couplings (RDC) are arranged in a circular helix manner and yet still conformed to an axially symmetric system used for deriving a generalized MAE formalism. 5 , 8 This developed new model is suitable not only for the ordered water trapped between the layers of myelin sheath but also for the $\mathrm{CH} 2$ groups of semisolid macromolecules in WM as demonstrated in Figure 7. 


\subsection{An angle offset $\varepsilon_{0}$ for "unbiased" orientation reference in vivo}

In general, the introduced angle shift $\varepsilon_{0}$ has significantly improved the fittings of documented anisotropic $R_{2}$ profiles. The fitted $\varepsilon_{0}$ appeared relatively increased in less anisotropic environments such as underdeveloped neonates and aging WM (Figs. 5A and 6F), in good agreement with the previous findings from DTI directional diffusivities. ${ }^{40,}{ }^{41}$ Specifically, a relatively increased $D_{\perp}$, equivalent to an increased $\varepsilon_{0}$ in our analysis (Figs. $2 \mathrm{D}$ and $6 \mathrm{~F}$ ), has been long before established as an imaging biomarker for demyelinating diseases. ${ }^{42}$

Usually, a predefined FA threshold was set to identify WM voxels in brain MR images, and an increasingly wider range of $\varepsilon_{0}$ angles would be expected within an image voxel when FA threshold decreased. As a result, an intrinsic $R_{2}$ or $R_{2}^{*}$ orientation dependence could be compromised as shown in Figure 7 when the FA threshold was set to a lower level. ${ }^{27}$ Accordingly, care should be exercised when comparing $R_{2}$ or $R_{2}^{*}$ orientation dependences from different studies if different FA thresholds were used. Of particularly importance is when an advanced fiber orientation extraction method rather than the standard DTI was employed. ${ }^{12}$

When the orientation $(\varepsilon)$ information was attained by physically rotating an ex vivo WM specimen relative to $B_{0}, \varepsilon_{0}$ merely indicates an initial sample angulation when the measurement started. For instance, as shown in the original Figure 2 from a prior $7 \mathrm{~T}$ study, ${ }^{18}$ the fitted $\varepsilon_{0}$ from two $R_{2}^{*}$ profiles (data not known) of WM (corpus callosum) ROIs delineated on one specimen (Sample 1) were $-28.8 \pm 1.4^{\circ}$ (green, ROI 1) and $31.4 \pm 1.2^{\circ}$ (magenta, ROI 2), respectively. The difference between these two angle offsets was about $120^{\circ}$ (i.e., $180^{\circ}-28.8^{\circ}-31.4^{\circ}$ ), close to an angle of $116^{\circ}$ measured using a protractor on the image.

\section{3 | Orientation-independent $R_{2}^{i}$ transverse relaxation}


An orientation-independent $R_{2}^{i}$ comprises not only an irreversible but also a reversible contribution. The irreversible part is largely induced by intrinsic molecular reorientations, increased with a lower temperature such as in situ as revealed in literarure. ${ }^{23}$ In contrast, the reversible mostly depends on $B_{0}$, primarily originating from chemical exchange (CHEX) and magnetic susceptibility effects. ${ }^{43}$ As demonstrated in Figs. 5A and 6C, $R_{2}^{i}$ was relatively reduced in underdeveloped and pathological WM compared to that in the healthy brain, consistent with decreased macromolecular content. Compared to those in vivo at $3 \mathrm{~T},{ }^{44} R_{2}^{i}$ became considerably larger ex vivo at $\geq 7 \mathrm{~T}$, due largely to an increased $B_{0}$ inhomogeneity at higher fields. Interestingly, $R_{2}^{i}$ from the sibling control group was relatively higher compared to that from the MS group (Figure 6C), which was ascribed to an excess iron deposition as a predisposition for $\mathrm{MS}^{25}$ As iron-induced transverse relaxation is orientation-independent, ${ }^{19}$ it is thus critical to accurately separate $R_{2}^{i}$ from $R_{2}^{a}$ for reliably evaluating an iron deposition in WM. ${ }^{45}$

\section{4 | Ordination-dependent $R_{2}^{a}$ transverse relaxation}

Both the amount of RDCs and the degrees of restricted reorientations govern the observed $R_{2}^{a} \cdot 3,5$

For the first cause, a relatively smaller $R_{2}^{a}$ was found in underdeveloped neonatal WM (Figure 5A) and compromised WM from people with MS (Figure 6B). Perhaps, an extreme "demyelination" case would be the elongated cellular constituents (e.g., microtubules and neurofilaments) in an intra-axonal space, revealing a considerably reduced $R_{2}^{a}$ compared to that in an extra-axonal space (data not shown). ${ }^{12}$ On the other hand, a considerably increased $R_{2}^{a}$ was measured at room temperatures on formalin-fixed WM specimens embedded in an agarose gel at higher fields $(\geq 7 \mathrm{~T}) .{ }^{44}$ Further, the measured $R_{2}^{a}$ from $\mathrm{CH} 2$ groups of semisolid macromolecules in WM was about $10^{4}$ times larger than that from dynamically ordered water close to the phospholipid bilayer surface (Table 3). 
Because the fitted $R_{2}^{i}$ and $R_{2}^{a}(\mathrm{FA}>0.7)$ from $\mathrm{CH} 2$ groups were comparable and the orientation-dependent function $f(\alpha, \varepsilon)$ maximizes up to 4 , an orientation-dependent myelinspecific contribution to the measured qMT signals could be estimated about $75 \%$, i.e., $4 R_{2}^{a} /\left(R_{2}^{i}+4 R_{2}^{a}\right) \times 100$. This finding agrees reasonably well with an earlier conclusion made from an MT-CPMG hybrid measurement of bovine optic nerve in that the MT effect from myelin water was roughly nine time larger than that for IE water. ${ }^{46}$ Hence, an orientationdependent $R_{2}$ profile derived from semisolid macromolecules in WM has provided a unique opportunity to quantify the qMT specificity to myelination.

\section{5 | A fitted $\alpha$ linked to myelination in WM}

The fitted $\alpha$ appears comparable $\left(\sim 70^{\circ}\right)$ in healthy human adult brain WM from different cohorts (Tables 1 and 3); in other words, an average thickness of myelin sheath encasing a typical axon is similar assuming a fixed axon radius. Particularly, one measure from Group D was derived from qMT (Figure 7), which was dramatically different from the standard $R_{2}$ or $R_{2}^{*}$ mapping. The qMT signals were largely linked to $\mathrm{CH} 2$ groups of semisolid lipid's long tails while the standard $R_{2}$ or $R_{2}^{*}$ mapping informed how water molecules were dynamically organized close to the surface of phospholipid bilayers in WM. The same fitting results from two markedly different measurements has provided a stringent test for the developed framework.

For neonates (Figure 5A) due to underdevelopment in WM and elderly groups (Figure 6E) due to aging or demyelination, the fitted $\alpha$ became smaller. This $\alpha$ tendency can be adequately accounted for, as demonstrated in Figure 3D, by a shrinking effective axon radius $r$ with a fixed helix pitch $2 \pi d$. Interestingly, analogous information can also be deduced from a well-established DTI-based myelin g-ratio, defined as the ratio of the inner to the outer diameter 
of the myelin sheath of a myelinated axon. ${ }^{47}$ In this regard, $\alpha$ could be considered as an alternative to the "global" g-ratio in WM but reveals an opposite correlation with myelination.

\subsection{An orientation-independent MR metric from an anisotropic $R_{2}$ in WM}

Even though the measured orientation-dependent $R_{2}$ or $R_{2}^{*}$ in $\mathrm{WM}$ can be adequately quantified with the proposed novel framework, the derived related anisotropic relaxation parameters remain non-localized by nature. To further exploit the orientation-dependent transverse relaxation as a reliable biomarker for myelin integrity on the voxel basis, the observed orientation dependences in $R_{2}$ or $R_{2}^{*}$ ought to be eliminated. To this end, quantitative $R_{1 \rho}$ dispersion imaging has been demonstrated for deriving an orientation-independent order parameter in articular cartilage; ${ }^{48,49}$ thus, more research is warranted in neuroimaging along the same line as done in musculoskeletal imaging.

\section{6 | CONCLUSIONS}

In summary, a biophysical model, embodied in a cylindrical helix, has been proposed to encompass both anisotropic translational and rotational water molecular motions in highly organized myelin microstructures in the human brain white matter. This developed model can better characterize the documented orientation-dependent transverse relaxation profiles from various physiological and pathological conditions, providing further insights into myelin microstructural alterations at the molecular level.

\section{ACKNOWLEDGEMENTS}

The author is very grateful to Dr. Harald E. Möller (Max Planck Institute for Human Cognitive and Brain Sciences, Leipzig, Germany) for his insightful comments, and to Dr. Vladimír 
Mlynárik (Medical University of Vienna, Vienna, Austria) for his valuable suggestions on the earlier versions of this manuscript. The author would also like to thank Dr. Enedino HernándezTorres (University of British Columbia, Vancouver, Canada) for sharing clinical anisotropic $R_{2}^{*}$ data in a public domain.

\section{DATA AVAILABILITY STATEMENT}

Data used in this work are available on request from the author.

\section{ORCID}

Yuxi Pang: https://orcid.org/0000-0001-5039-0236

\section{REFERENCES}

1. Bydder M, Rahal A, Fullerton GD, Bydder GM. The magic angle effect: a source of artifact, determinant of image contrast, and technique for imaging. J Magn Reson Imaging. Feb 2007;25(2):290-300. doi:10.1002/jmri.20850

2. Fullerton GD. The magic angle effect in NMR and MRI of cartilage. In: Xia Y, Momot $\mathrm{KI}$, eds. Biophysics and Biochemistry of Cartilage by NMR and MRI. The Royal Society of Chemistry; 2016:109-144.

3. Momot KI, Pope JM, Wellard RM. Anisotropy of spin relaxation of water protons in cartilage and tendon. NMR Biomed. Apr 2010;23(3):313-24. doi:10.1002/nbm.1466

4. Peto S, Gillis P, Henri VP. Structure and dynamics of water in tendon from NMR relaxation measurements. Biophysical journal. 1990;57(1):71-84.

5. Berendsen HJC. Nuclear magnetic resonance study of collagen hydration. J Chem Phys. 1962;36(12):3297-3305. doi:Doi 10.1063/1.1732460

6. Tourell MC, Momot KI. Molecular dynamics of a hydrated collagen peptide: insights into rotational motion and residence times of single-water bridges in collagen. $J$ Phys Chem B. Dec 15 2016;120(49):12432-12443. doi:10.1021/acs.jpcb.6b08499

7. Grunder W. MRI assessment of cartilage ultrastructure. NMR Biomed. Nov 2006;19(7):855-76. doi:10.1002/nbm.1092

8. Pang Y. Characterization of anisotropic T2W signals from human knee femoral cartilage: The magic angle effect on a spherical surface. NMR in Biomedicine. 2021:e4535.

9. Chappell KE, Robson MD, Stonebridge-Foster A, et al. Magic angle effects in MR neurography. AJNR Am J Neuroradiol. Mar 2004;25(3):431-40. 
10. Lee J, Shin H-G, Jung W, Nam Y, Oh S-H, Lee J. An R2* model of white matter for fiber orientation and myelin concentration. Neuroimage. 2017;162:269-275.

11. Knight MJ, Dillon S, Jarutyte L, Kauppinen RA. Magnetic resonance relaxation anisotropy: Physical principles and uses in microstructure imaging. Biophysical journal. 2017;112(7):1517-1528.

12. Tax CMW, Kleban E, Chamberland M, Baraković M, Rudrapatna U, Jones DK. Measuring compartmental T(2)-orientational dependence in human brain white matter using a tiltable RF coil and diffusion-T(2) correlation MRI. Neuroimage. Apr 29 2021;236:117967. doi:10.1016/j.neuroimage.2021.117967

13. Knight MJ, Wood B, Couthard E, Kauppinen R. Anisotropy of spin-echo T2 relaxation by magnetic resonance imaging in the human brain in vivo. Biomedical Spectroscopy and Imaging. 2015;4(3):299-310.

14. Birkl C, Doucette J, Fan M, Hernandez-Torres E, Rauscher A. Myelin water imaging depends on white matter fiber orientation in the human brain. Magn Reson Med. 2021;85(4):2221-2231.

15. Gil R, Khabipova D, Zwiers M, Hilbert T, Kober T, Marques JP. An in vivo study of the orientation - dependent and independent components of transverse relaxation rates in white matter. NMR in Biomedicine. 2016;29(12):1780-1790.

16. Bender B, Klose U. The in vivo influence of white matter fiber orientation towards B0 on T2* in the human brain. NMR in biomedicine. 2010;23(9):1071-1076.

17. Cherubini A, Péran P, Hagberg GE, et al. Characterization of white matter fiber bundles with T2* relaxometry and diffusion tensor imaging. Magn Reson Med. 2009;61(5):1066-1072.

18. Lee J, van Gelderen P, Kuo L-W, Merkle H, Silva AC, Duyn JH. T2*-based fiber orientation mapping. Neuroimage. 2011;57(1):225-234.

19. Oh S-H, Kim Y-B, Cho Z-H, Lee J. Origin of B0 orientation dependent R2*(= 1/T2*) in white matter. Neuroimage. 2013;73:71-79.

20. Lee J, Nam Y, Choi JY, Kim EY, Oh SH, Kim DH. Mechanisms of T2* anisotropy and gradient echo myelin water imaging. NMR in Biomedicine. 2017;30(4):e3513.

21. Wharton S, Bowtell R. Gradient echo based fiber orientation mapping using R2* and frequency difference measurements. Neuroimage. 2013;83:1011-1023.

22. Wharton S, Bowtell R. Fiber orientation-dependent white matter contrast in gradient echo MRI. Proceedings of the National Academy of Sciences. 2012;109(45):18559-18564.

23. Lenz C, Berger C, Bauer M, Scheurer E, Birkl C. Sensitivity of fiber orientation dependent $\mathrm{R} 2 *$ to temperature and post mortem interval. Magn Reson Med. Jun 4 2021;doi:10.1002/mrm.28874

24. Weber AM, Zhang Y, Kames C, Rauscher A. Myelin water imaging and R2* mapping in neonates: Investigating R2* dependence on myelin and fibre orientation in whole brain white matter. NMR in Biomedicine. 2020;33(3):e4222.

25. Hernández-Torres E, Wiggermann V, Hametner S, et al. Orientation dependent MR signal decay differentiates between people with MS, their asymptomatic siblings and unrelated healthy controls. Plos One. 2015;10(10):e0140956.

26. Cheng J-X, Pautot S, Weitz DA, Xie XS. Ordering of water molecules between phospholipid bilayers visualized by coherent anti-Stokes Raman scattering microscopy. Proceedings of the National Academy of Sciences. 2003;100(17):9826-9830.

27. Pampel A, Müller DK, Anwander A, Marschner H, Möller HE. Orientation dependence of magnetization transfer parameters in human white matter. Neuroimage. 2015;114:136-146. 
28. Denk C, Torres EH, MacKay A, Rauscher A. The influence of white matter fibre orientation on MR signal phase and decay. NMR in Biomedicine. 2011;24(3):246-252.

29. Wennerström H. Proton nuclear magnetic resonance lineshapes in lamellar liquid crystals. Chemical Physics Letters. 1973;18(1):41-44.

30. Bloom M, Burnell EE, MacKay AL, Nichol CP, Valic MI, Weeks G. Fatty acyl chain order in lecithin model membranes determined from proton magnetic resonance. Biochemistry. 1978;17(26):5750-5762.

31. Simons M, Nave K-A. Oligodendrocytes: myelination and axonal support. Cold Spring Harbor perspectives in biology. 2016;8(1):a020479.

32. Callaghan P, Jolley K, Lelievre J. Diffusion of water in the endosperm tissue of wheat grains as studied by pulsed field gradient nuclear magnetic resonance. Biophysical journal. 1979;28(1):133-141.

33. Krasnosselskaia LV, Fullerton GD, Dodd SJ, Cameron IL. Water in tendon: orientational analysis of the free induction decay. Magn Reson Med. 2005;54(2):280-288.

34. Mädler B, Drabycz SA, Kolind SH, Whittall KP, MacKay AL. Is diffusion anisotropy an accurate monitor of myelination?: Correlation of multicomponent T2 relaxation and diffusion tensor anisotropy in human brain. Magnetic resonance imaging. 2008;26(7):874-888.

35. Bevington PR, Robinson DK. Data reduction and error analysis for the physical sciences. 3rd ed. McGraw-Hill; 2003:xi, 320 p.

36. Basser PJ, Pierpaoli C. Microstructural and physiological features of tissues elucidated by quantitative-diffusion-tensor MRI. J Magn Reson. 2011;213(2):560-570.

37. Markwardt CB. "Non-linear least-squares fitting in IDL with MPFIT", in proc. Astronomical Data Analysis Software and Systems XVIII, Quebec, Canada, ASP Conference Series, Vol. 411, eds. D. Bohlender, P. Dowler \& D. Durand (Astronomical Society of the Pacific: San Francisco), p. 251-254. 2009:

38. Ahearn TS, Staff RT, Redpath TW, Semple SIK. The use of the Levenberg-Marquardt curve-fitting algorithm in pharmacokinetic modelling of DCE-MRI data. Physics in Medicine \& Biology. 2005;50(9):N85.

39. Selinger JV, Spector MS, Schnur JM. Theory of self-assembled tubules and helical ribbons. ACS Publications; 2001.

40. Song S-K, Sun S-W, Ramsbottom MJ, Chang C, Russell J, Cross AH. Dysmyelination revealed through MRI as increased radial (but unchanged axial) diffusion of water. Neuroimage. 2002;17(3):1429-1436.

41. Beaulieu C. The biological basis of diffusion anisotropy. Diffusion MRI. Elsevier; 2014:155-183.

42. Aung WY, Mar S, Benzinger TL. Diffusion tensor MRI as a biomarker in axonal and myelin damage. Imaging in medicine. 2013;5(5):427.

43. Mitsumori F, Watanabe H, Takaya N, et al. Toward understanding transverse relaxation in human brain through its field dependence. Magnet Reson Med. 2012;68(3):947-953.

44. Pang Y. Anisotropic transverse relaxation in the human brain white matter induced by restricted rotational diffusion. In: Proceedings of the 29th Virtual Annual Meeting of ISMRM, 2021. (abstract: 1711):

45. Möller HE, Bossoni L, Connor JR, et al. Iron, myelin, and the brain: neuroimaging meets neurobiology. Trends in neurosciences. 2019;42(6):384-401. 
46. Stanisz GJ, Kecojevic A, Bronskill M, Henkelman R. Characterizing white matter with magnetization transfer and T2. Magnetic Resonance in Medicine: An Official Journal of the International Society for Magnetic Resonance in Medicine. 1999;42(6):1128-1136.

47. Stikov N, Campbell JS, Stroh T, et al. In vivo histology of the myelin g-ratio with magnetic resonance imaging. Neuroimage. 2015;118:397-405.

48. $\quad$ Pang Y. An order parameter without magic angle effect (OPTIMA) derived from R1 $\rho$ dispersion in ordered tissue. Magn Reson Med. 2020;83(5):1783-1795. doi:10.1002/mrm.28045

49. Pang Y, Palmieri - Smith RM, Maerz T. An efficient R1 $\rho$ dispersion imaging method for human knee cartilage using constant magnetization prepared turbo-FLASH. NMR in Biomedicine. 2021:e4500.

\section{SEVEN FIGURE CAPTIONS}

(A)

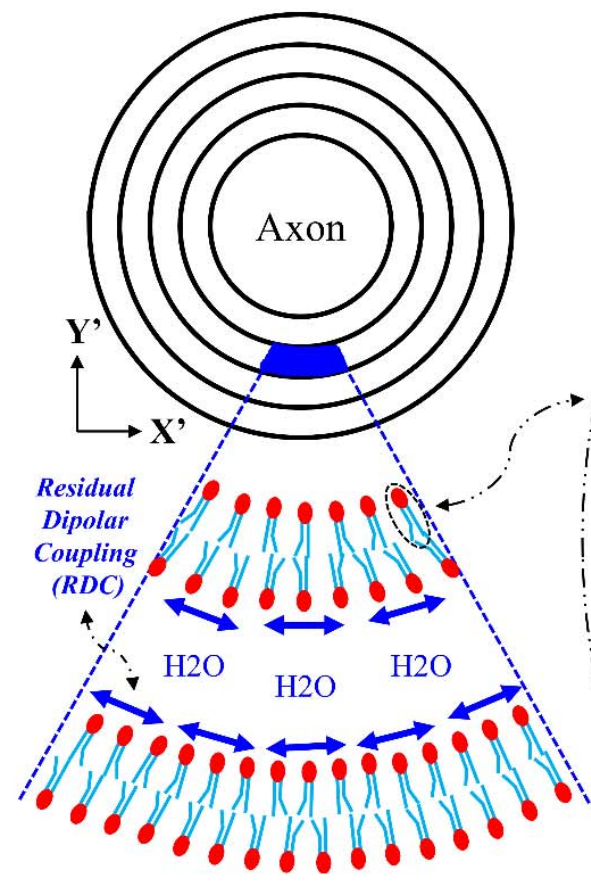

(B)

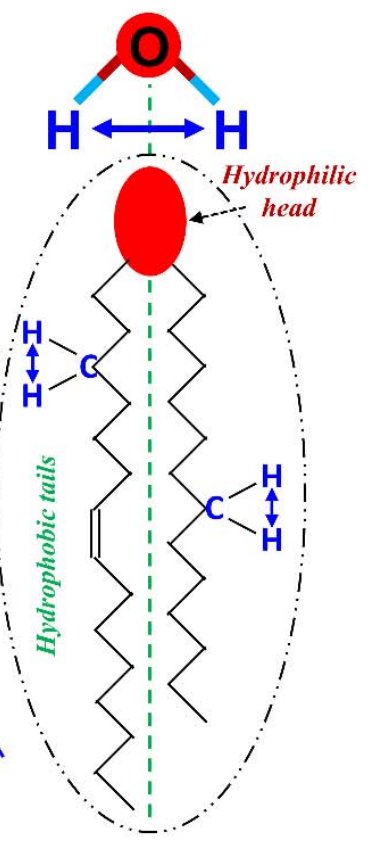

(C)

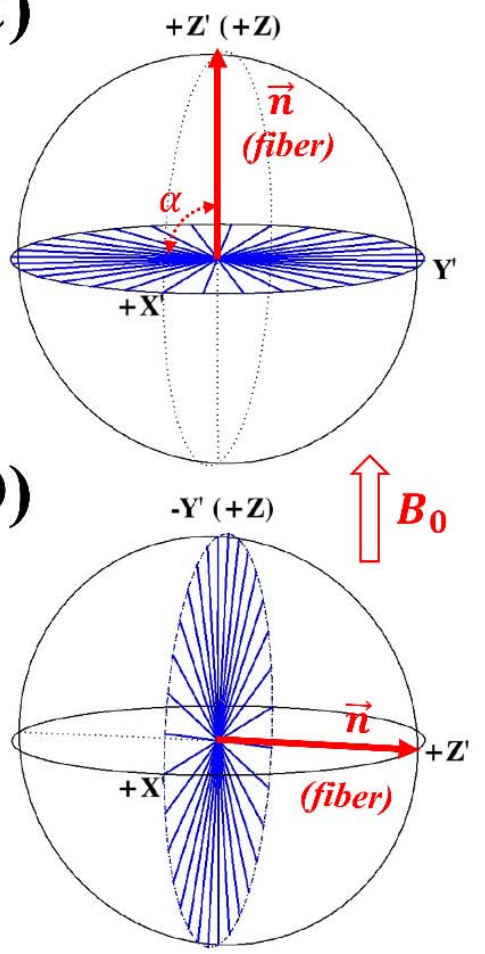


FIGURE 1. Schematics of residual dipolar coupling (RDC, blue double-headed arrows) organized around an axon fiber (A). A lipid molecule is highlighted (B) with RDCs of ordered water and methylene $(\mathrm{CH} 2)$ groups respectively perpendicular to and parallel to the direction of hydrophobic tails (green dashed line). An axially symmetric model of RDCs (blue lines) with an axon direction $\vec{n}$ (in the molecular reference frame, $\mathrm{X}^{\prime} \mathrm{Y}^{\prime} \mathrm{Z}^{\prime}$ ) parallel to (C) and perpendicular to (D) the main magnetic field $B_{0}$ (in the laboratory reference frame, XYZ). A representative $<\mathrm{H}-$ $\mathrm{H}>$ vector makes an angle $\alpha=90^{\circ}$ to $\vec{n}$. Figures $1 \mathrm{~A}$ and $1 \mathrm{~B}$ were adapted from Figure 2 in the reference. $^{26}$

(A)

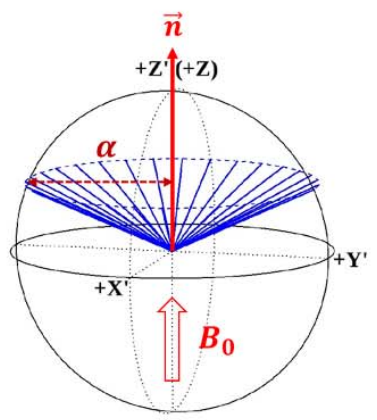

Anisotropic rotational diffusion
(B)

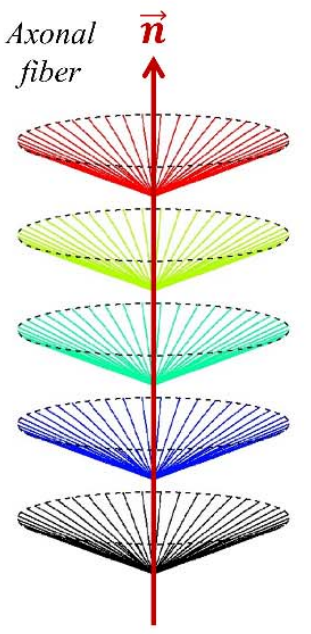

(C)

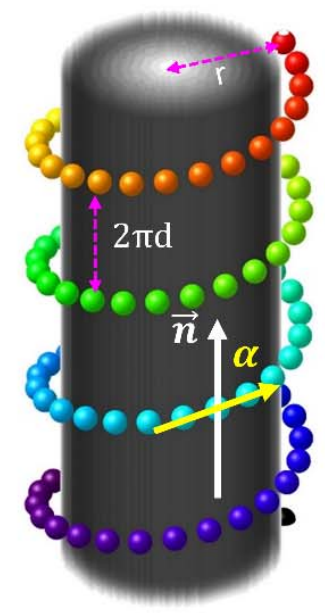

(D)

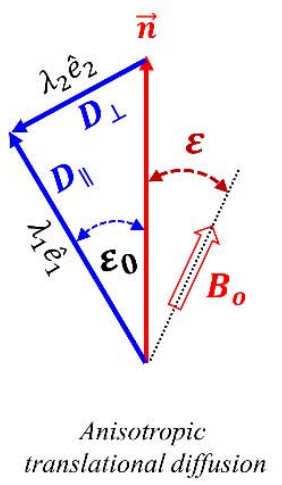

FIGURE 2. A stack of axially symmetric models (B) in space can be characterized by a representative funnel model with an open angle $\alpha$ (A), which in turn is mathematically equivalent to a cylindrical helix (C) for both an anisotropic molecular rotational motion around the helix primary axis $(\vec{n})$ and an anisotropic molecular translational diffusion along $\vec{n}$ (D). The colored balls denote the representative hydrophilic heads of lipid bilayers, and a typical RDC 
along helix tangent line (yellow) makes a constant angle $(\alpha)$ with $\vec{n}$ (white), Note, the direction of axial diffusivity $\left(D_{\|}\right)$deviates an angle $\varepsilon_{0}$ from the direction of anisotropic translational diffusion along $\vec{n}$.
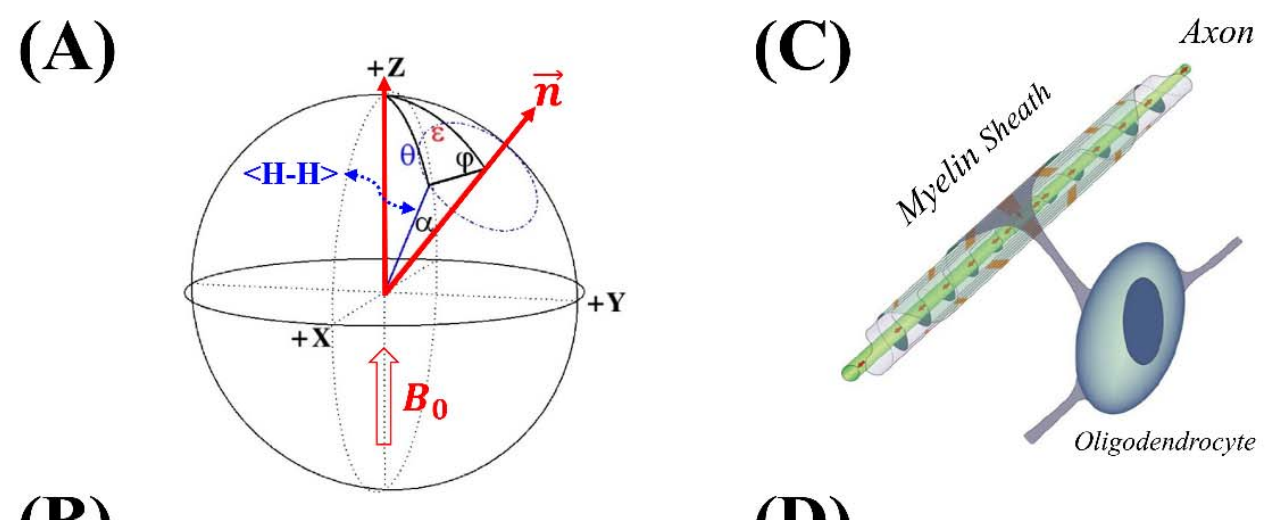

(B)

\section{(D)}
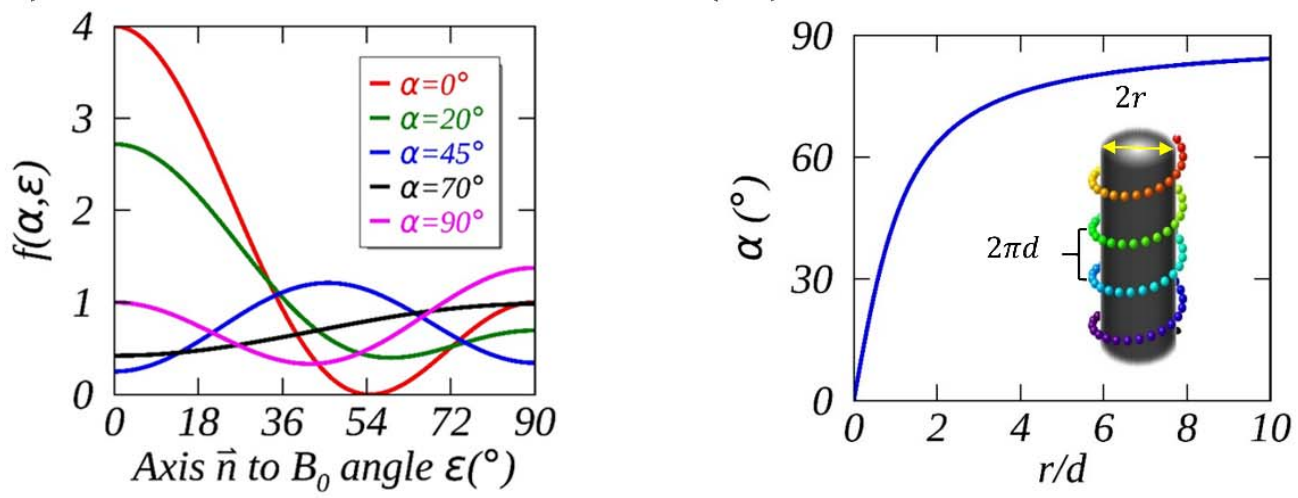

FIGURE 3. An axially symmetric model (A) in the laboratory reference frame (XYZ) for deriving anisotropic transverse relaxation orientation dependences $f(\alpha, \varepsilon)$ as profiled for five $\alpha$ angles from $0^{\circ}$ to $90^{\circ}$ (B). A schematic representation (adapted from Figure 3 in the reference ${ }^{31}$ ) is shown for spiral myelination around an axon (C). A mathematical relationship between angle $\alpha$ and $r / d$ is plotted, with $r$ and $2 \pi d$ standing for an effective axon radius and a helix pitch, respectively (D). 

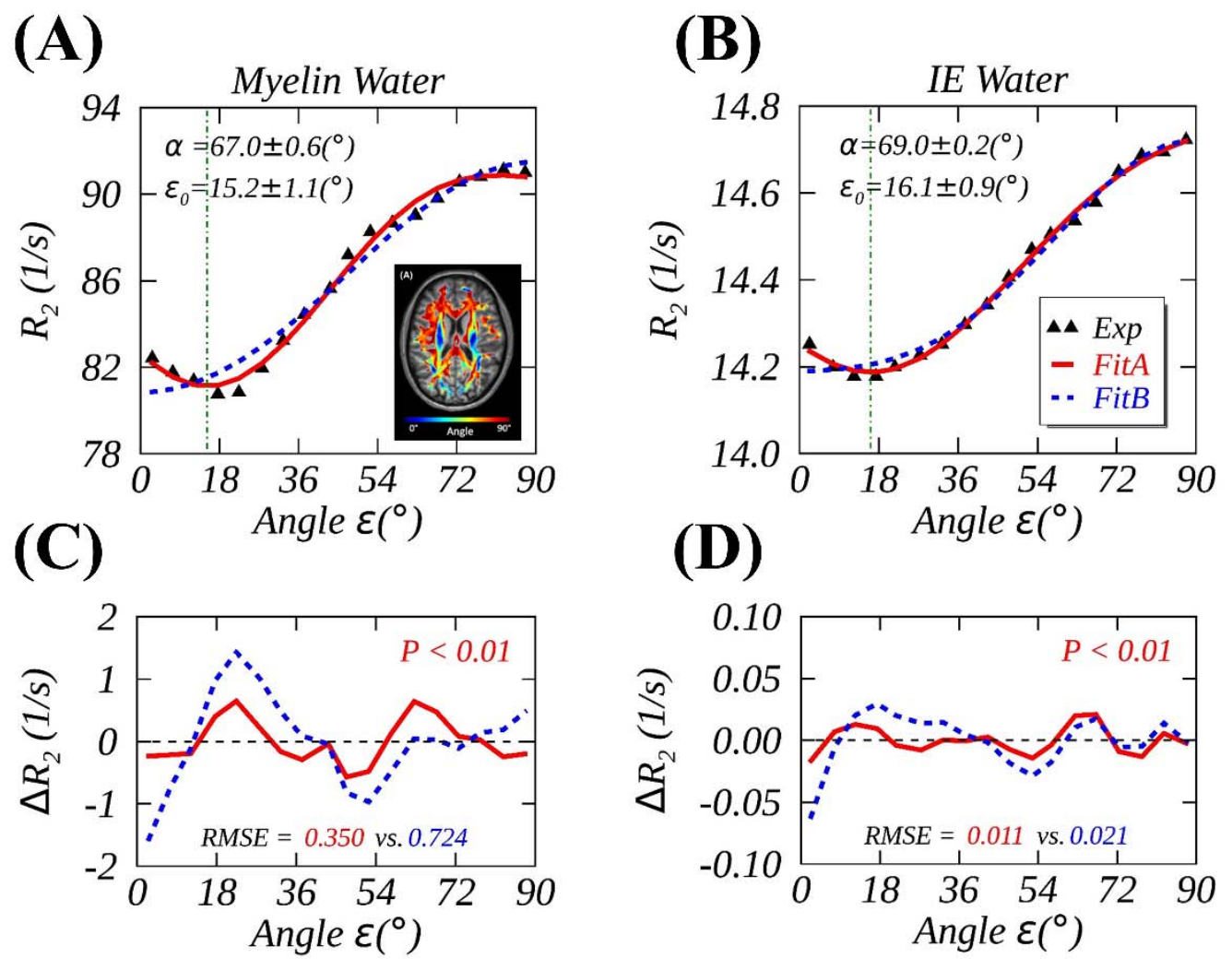

FIGURE 4. Measured (black triangles) and fitted (Fit A, red solid lines; Fit B, blue dashed lines) anisotropic $R_{2}$ of myelin water (A) and intra- and extracellular (IE) water (B) in the human brain WM in vivo at 3T, with the corresponding fitting residues $\Delta R_{2}=$ Fitted-Measured $(\mathrm{C}$ and D). The measured data and an inset image (A) were retrieved and adapted from the reference. ${ }^{14}$ 

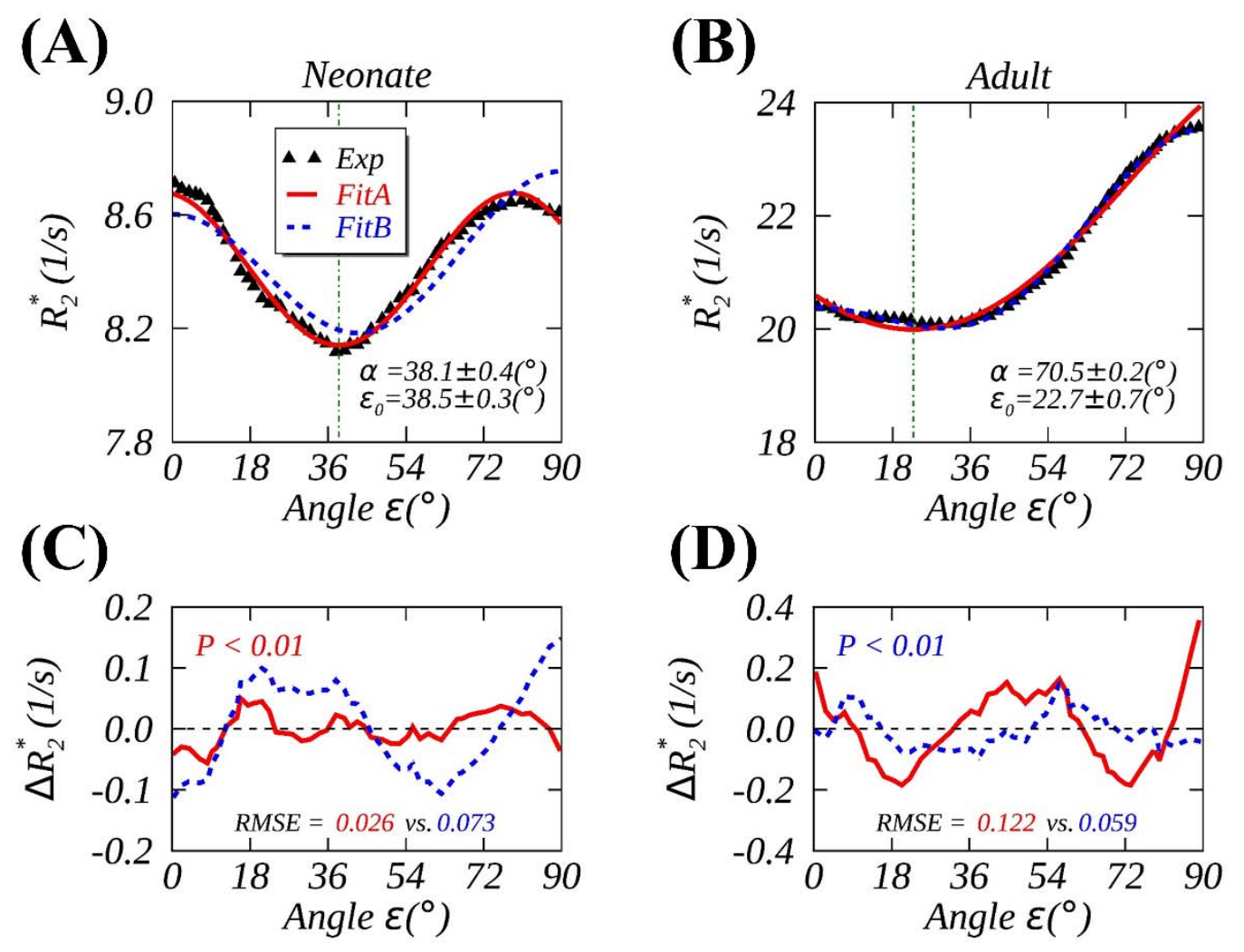

FIGURE 5. Measured (black triangles) and fitted (Fit A, red solid lines; Fit B, blue dashed lines) anisotropic $R_{2}^{*}$ in WM of neonates (A) and healthy adults (B) in vivo at 3T, with the corresponding fitting residues $\Delta R_{2}=$ Fitted-Measured $(\mathrm{C}$ and $\mathrm{D})$. The measured data were retrieved from the reference. ${ }^{24}$ 
(A)

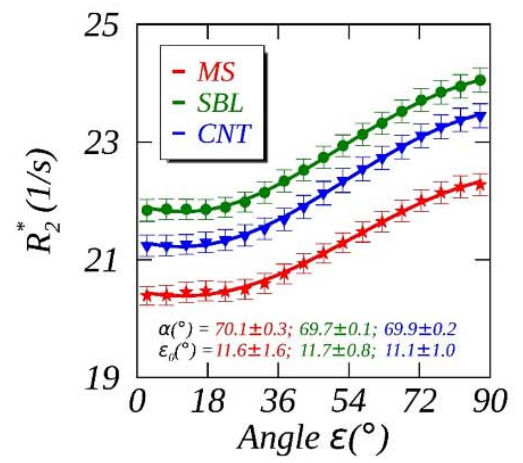

(B)

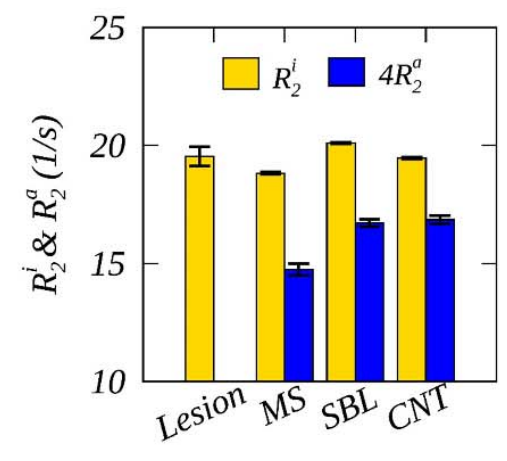

(C)

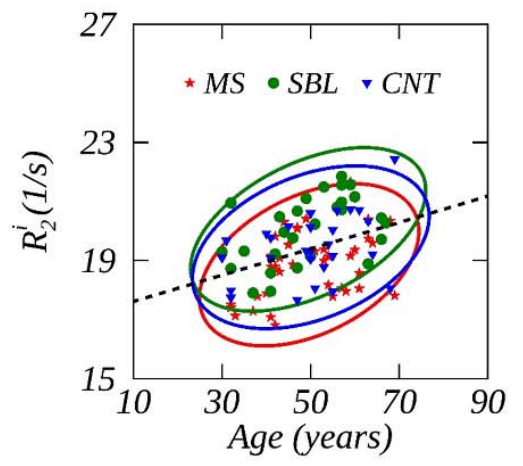

(D)

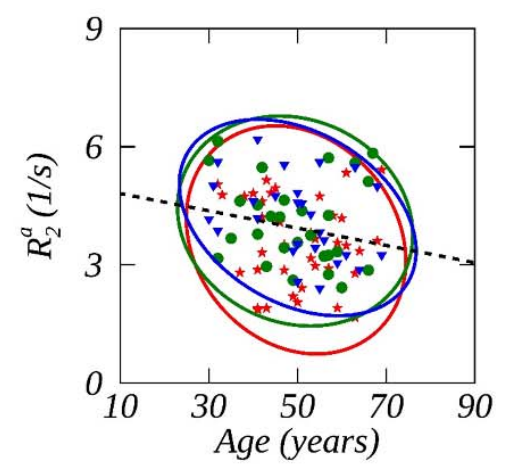

(E)

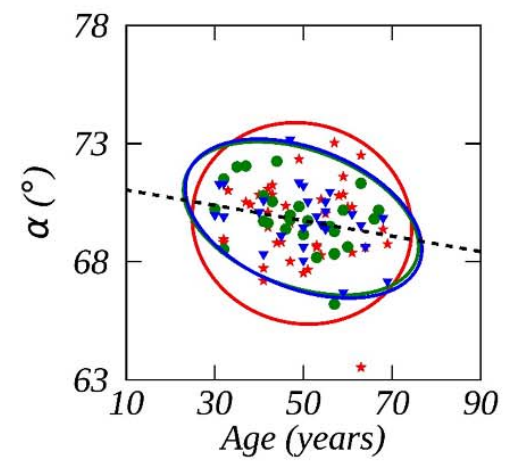

(F)

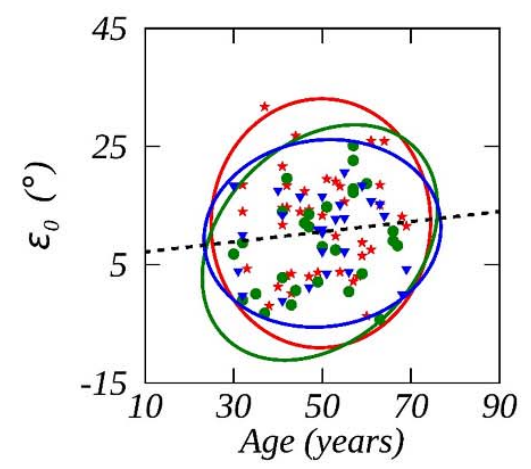

FIGURE 6. Measured (symbols) and fitted (Fit A, lines) average anisotropic $R_{2}^{*}$ in WM from people with multiple sclerosis (MS, red) and two controls of age-matched asymptomatic siblings (SBL, green) and unrelated healthy subjects (CNT, blue), with the fitted $\alpha$ and $\varepsilon_{0}$ imprinted in colors (A). The fitted $R_{2}^{i}$ and $R_{2}^{a}$ from the average $R_{2}^{*}$ profiles are shown for three cohorts as well as lesions (B). Correlations between the fits from individual subjects and their ages are depicted for $R_{2}^{i}(\mathrm{C}), R_{2}^{a}(\mathrm{D}), \alpha(\mathrm{E})$, and $\varepsilon_{0}(\mathrm{~F})$, overlaid with $95 \%$ confidence ellipses from individual subgroups and linear regression lines (black dashed lines) from combined subgroups. The measured $R_{2}^{*}$ data were retrieved from Supporting Information in the reference. ${ }^{25}$ 
(A)

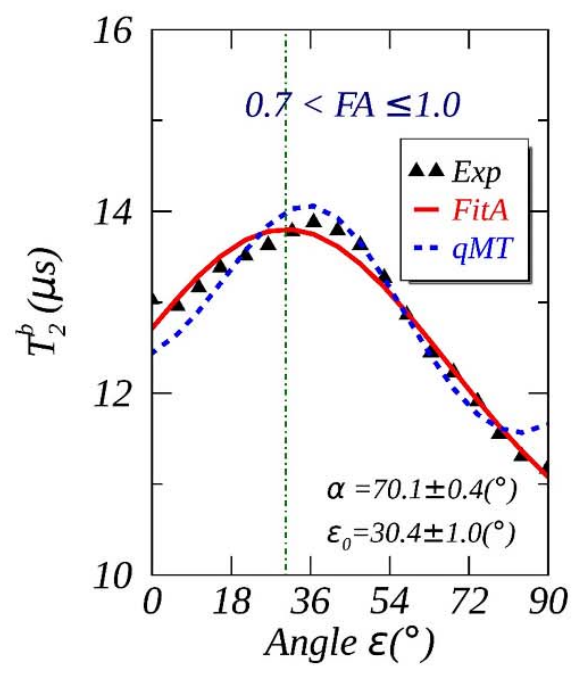

(B)

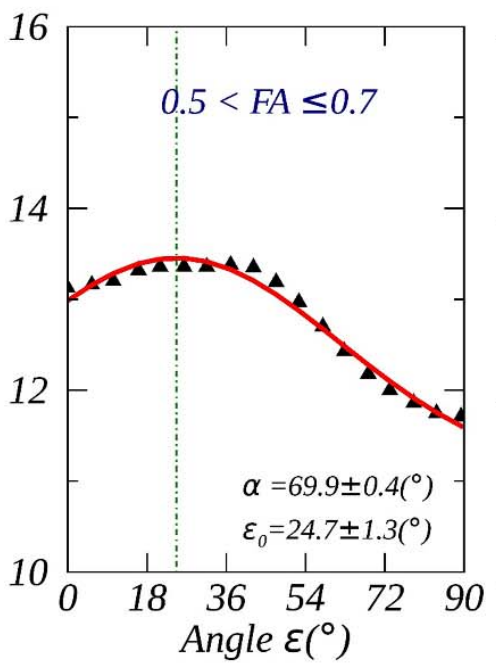

(C)

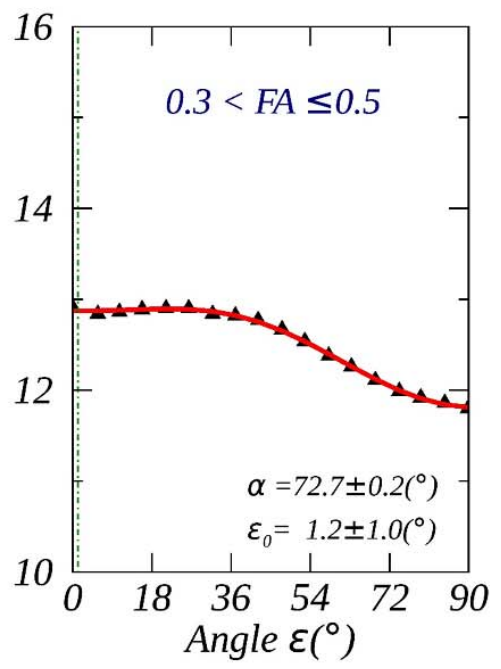

FIGURE 7. Measured (black triangles) and fitted (Fit A, red solid lines) $T_{2}^{b}$ of semisolid macromolecular in WM based on quantitative magnetization transfer imaging in vivo at 3T. The orientation-dependent $T_{2}^{b}$ profiles were obtained from image voxels with diffusion fractional anisotropy (FA) threshold respectively set to the ranges of $[0.7,1.0)(\mathrm{A}),[0.5,0.7)(\mathrm{B})$ and $[0.3$, 0.5) (C). An alternative fit to Fit A with $\alpha=90^{\circ}$ and $\varepsilon_{0}=0^{\circ}$ (qMT, blue dashed line) is shown for $T_{2}^{b}$ data with FA $>0.7$. The measured $T_{2}^{b}$ data were retrieved from the reference. ${ }^{27}$ 


\section{THREE TABLES}

TABLE 1. The fits from Fit A for the documented anisotropic $R_{2}$ and $R_{2}^{*}$ and related DTI metrics in WM. Data are means \pm standard deviations except for one group age indicated by mean and range (square brackets).

\begin{tabular}{|c|c|c|c|c|c|c|c|c|c|c|}
\hline $\begin{array}{l}\text { Group } \\
\text { ID }\end{array}$ & $\begin{array}{l}\text { Data } \\
\text { (n) }\end{array}$ & $\begin{array}{l}\text { Age } \\
\text { (yrs) }\end{array}$ & $\begin{array}{c}R_{2}^{i} \\
(1 / \mathrm{s})\end{array}$ & $\begin{array}{c}R_{2}^{a} \\
(1 / \mathrm{s})\end{array}$ & $\alpha\left(^{\circ}\right)$ & $\varepsilon_{0}\left({ }^{\circ}\right)$ & $\begin{array}{c}D_{\|} \\
\left(\mu \mathrm{m}^{2} / \mathrm{ms}\right)\end{array}$ & $\begin{array}{c}D_{\perp} \\
\left(\mu \mathrm{m}^{2} / \mathrm{ms}\right)\end{array}$ & FA & $\begin{array}{c}\text { DTI } \\
\varepsilon_{0}\left({ }^{\circ}\right)\end{array}$ \\
\hline \multirow{2}{*}{ A } & Myelin (8) & \multirow{2}{*}{$\begin{array}{c}26.0 \\
{[21-33]}\end{array}$} & $76.5 \pm 0.6$ & $16.0 \pm 0.8$ & $67.0 \pm 0.6$ & $15.2 \pm 1.1$ & \multirow{2}{*}{$1.44 \pm 0.24$} & \multirow{2}{*}{$0.47 \pm 0.11$} & \multirow{2}{*}{$0.60 \pm 0.12$} & \multirow{2}{*}{$16.7 \pm 6.1$} \\
\hline & IE (8) & & $13.8 \pm 0.0$ & $1.0 \pm 0.0$ & $69.0 \pm 0.2$ & $16.1 \pm 0.9$ & & & & \\
\hline \multirow{2}{*}{ B } & Neonates (8) & $0.78 \pm 0.02$ & $7.2 \pm 0.1$ & $1.3 \pm 0.1$ & $38.1 \pm 0.4$ & $38.5 \pm 0.3$ & $1.69 \pm 0.03$ & $1.06 \pm 0.04$ & $0.31 \pm 0.01$ & $32.1 \pm 1.1$ \\
\hline & Adults (5) & $31.2 \pm 8.3$ & $16.1 \pm 0.1$ & $8.7 \pm 0.2$ & $70.5 \pm 0.2$ & $22.7 \pm 0.7$ & $1.24 \pm 0.03$ & $0.51 \pm 0.04$ & $0.53 \pm 0.03$ & $22.4 \pm 1.7$ \\
\hline \multirow{3}{*}{ C } & MS (39) & $49.7 \pm 10.1$ & $18.9 \pm 1.1$ & $3.6 \pm 1.2$ & $69.6 \pm 1.7$ & $12.0 \pm 8.6$ & $\mathrm{n} / \mathrm{a}$ & $\mathrm{n} / \mathrm{a}$ & $\mathrm{n} / \mathrm{a}$ & $\mathrm{n} / \mathrm{a}$ \\
\hline & SBL (28) & $49.5 \pm 10.5$ & $20.1 \pm 1.1$ & $4.1 \pm 1.1$ & $69.9 \pm 1.3$ & $8.8 \pm 8.1$ & $\mathrm{n} / \mathrm{a}$ & $\mathrm{n} / \mathrm{a}$ & $\mathrm{n} / \mathrm{a}$ & $\mathrm{n} / \mathrm{a}$ \\
\hline & CNT (27) & $50.0 \pm 10.7$ & $19.5 \pm 1.1$ & $4.2 \pm 1.0$ & $69.8 \pm 1.4$ & $10.3 \pm 6.5$ & $\mathrm{n} / \mathrm{a}$ & $\mathrm{n} / \mathrm{a}$ & $\mathrm{n} / \mathrm{a}$ & $\mathrm{n} / \mathrm{a}$ \\
\hline
\end{tabular}


TABLE 2. Pearson correlation coefficients (PCC) between the fits (Fit A) of anisotropic $R_{2}^{*}$ in WM and the ages of people from individual or combined groups. The statistical significances of the correlation are indicated by $P$-values. MS, multiple sclerosis; SBL, age-matched asymptomatic siblings; CNT, age-matched unrelated healthy subjects.

\begin{tabular}{|c|c|c|c|c|c|c|c|c|}
\hline \multirow{2}{*}{ Fits } & \multicolumn{2}{|c|}{ MS } & \multicolumn{2}{|c|}{ SBL } & \multicolumn{2}{|c|}{ CNT } & \multicolumn{2}{|c|}{ ALL } \\
\hline & PCC & $\mathrm{P}$-value & PCC & P-value & PCC & P-value & PCC & P-value \\
\hline$R_{2}^{i}$ & 0.41 & 0.01 & 0.48 & 0.01 & 0.38 & 0.05 & 0.38 & $<0.01$ \\
\hline$R_{2}^{a}$ & -0.19 & 0.25 & -0.13 & 0.51 & -0.34 & 0.08 & -0.20 & 0.05 \\
\hline$\alpha$ & -0.06 & 0.72 & -0.37 & 0.05 & -0.38 & 0.05 & -0.23 & 0.03 \\
\hline$\varepsilon_{0}$ & 0.01 & 0.96 & 0.30 & 0.12 & 0.07 & 0.75 & 0.11 & 0.28 \\
\hline
\end{tabular}

TABLE 3. The fits from Fit A for the documented anisotropic $T_{2}^{b}$ profiles of semisolid macromolecules in WM obtained from quantitative magnetization transfer imaging in vivo at 3T. The diffusion fractional anisotropy (FA) threshold ranges were also provided. The fits are means \pm standard deviations

\begin{tabular}{|c|c|c|c|c|c|c|}
\hline $\begin{array}{c}\text { Group D } \\
\text { Data (n) }\end{array}$ & $\begin{array}{c}\text { Age } \\
(\mathrm{yrs})\end{array}$ & $\begin{array}{c}\boldsymbol{R}_{\mathbf{2}}^{\boldsymbol{i}} \\
\left(10^{3} 1 / \mathrm{s}\right)\end{array}$ & $\begin{array}{c}\boldsymbol{R}_{2}^{\boldsymbol{\alpha}} \\
\left(10^{3} 1 / \mathrm{s}\right)\end{array}$ & $\boldsymbol{\alpha}\left(^{\circ}\right)$ & $\boldsymbol{\varepsilon}_{\mathbf{0}}\left({ }^{\circ}\right)$ & FA \\
\hline & & $54.4 \pm 2.1$ & $42.5 \pm 3.6$ & $70.1 \pm 0.4$ & $30.4 \pm 1.0$ & $(0.7,1.0]$ \\
\hline $\begin{array}{c}\text { Adults } \\
(7)\end{array}$ & 26.0 & $63.8 \pm 1.0$ & $25.3 \pm 1.7$ & $69.9 \pm 0.4$ & $24.7 \pm 1.3$ & $(0.5,0.7]$ \\
& {$[19-33]$} & $70.6 \pm 0.1$ & $13.2 \pm 0.2$ & $72.7 \pm 0.2$ & $1.2 \pm 1.0$ & $(0.3,0.5]$ \\
\hline
\end{tabular}

